\title{
Perifrasi verbali con eỉuí più participio nelle varianti del Pentateuco dei LXX
}

\author{
[Perífrasis verbales con cỉuí más participio en las variantes del \\ Pentateuco de los LXX] \\ Rosario PIERRI \\ Faculty of Biblical Sciences and Archaeology (SBF, Jerusalem) \\ rosario.pierri@studiumbiblicum.org
}

Resumen: Nell'articolo sono raccolte e discusse le varianti con zỉjí più participio individuate nei testimoni della tradizione testuale del Pentateuco dei LXX; sono considerate anche le varianti con yívoual ma solo quelle concerneti il predicato nominale. La finalità è di osservare se vi siano tracce dell'affermazione della perifrasi nelle varianti, eventuali concentrazioni di frequenza insieme a sfumature d'uso, con particolare attenzione alla datazione dei testimoni dove appaiono. Un paragrafo è riservato anche alle varianti di costruzioni con predicato nominale (copula + aggettivo) rispetto a un verbo finito.

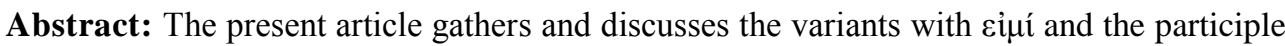
found in the testimonies of the textual traditions of the Pentateuch of the Lxx. They are also considered the variants with yivouar but only those concerning the nominal predicate. The purpose is to observe if there are traces of the affirmation of the periphrasis in the variants, perhaps concentrations of frequency together with undertones of use, with special attention to the dating of the testimonies where they are to be found. One paragraph is reserved to the variants of construction with the nominal predicate (copula + adjective) with respect to a finite verb.
\end{abstract}

Palabras clave: Perífrasis. Participios. Auxiliar. Cópula. Agentivo. Diatesis. Variantes.

Key words: Periphrasis. Participles. Auxiliary. Copula. Agentive. Diathesis. Variants.

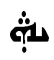


Questa ricerca si collega a un contributo sulle perifrasi verbali composte da yívo $\mu \alpha 1$ ed ह̌ $\sigma \tau \kappa \kappa \alpha$ e participio, che ho pubblicato di recente, ${ }^{1}$ nel quale per l'approfondimento della costruzione ho dato spazio soprattutto alla perifrasi composta da eíú più participio. ${ }^{2}$

Si può dire che in quel saggio la discussione si è articolata tra due poli: la comparsa della perifrasi verbale e la sua natura. Quanto al primo punto, gli autori discutono in che misura essa rispecchi un fenomeno interno alla lingua greca o subisca influssi esterni, soprattutto dall'aramaico o dall'ebraico. ${ }^{3}$ Una volta stabilito che - e appare la teoria più probabile - la costruzione si sia affermata

1 R. PIERRI, "Perifrasi verbali con $\gamma$ ívo $\mu \alpha$ ed $\check{\sigma} \sigma \tau \eta \kappa \alpha$ nei LXX", in G. GEIGER (a cura di, -

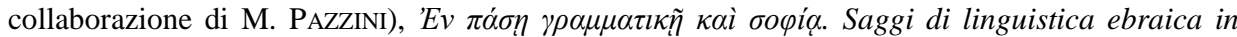
onore di Alviero Niccacci ofm, «SBF Analecta» 78 (Gerusalemme - Milano 2011), pp. 295-336.

2 L'attenzione al verbo eỉ $\mu$ í non è stata casuale. Qui è sufficiente citare qualche autore. Per S.E. PORTER, Verbal Aspect in the Greek of the New Testament, with Reference to Tense and Mood, «Studies in Biblical Greek» 1 (New York, 1989), p. 449: "There are a number of constructions in

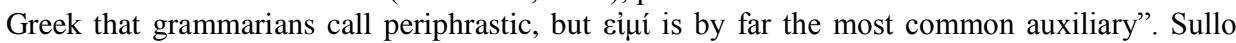
sviluppo della perifrasi con il perfetto con i verbi essere e avere nelle lingue europee cf. B. DRINKA, "The Formation of Periphrastic Perfects and Passives in Europe: An Areal Approach", in B.J. BlAKE - K. BuRRIDGE (ed.), Historical Linguistics 2001 (Amsterdam - Philadelphia, 2003), pp. 105-128; T. Evans, "Periphrastic Tense Forms in the Greek of Tobit", in F. García MARTÍNEZ - M. VERVENNE (ed.), Interpreting Translation: Studies on the LXX and Ezekiel in Honour of Johan Lust, «Bibliotheca Ephemeridum Theologicarum Lovaniensium» 192 (Leuven, 2005), p. 112 nota 21 si dice che le perifrasi con $\gamma^{\prime}(\gamma) v o \mu \alpha$ e vं $\pi \alpha ́ \rho \chi \omega$ molto probabilmente ("arguably") sono variazioni di cỉú più participio, almeno nel periodo della koinè.

3 L'affermazione delle perifrasi verbali è stata oggetto di studi che hanno interessato anche la lingua greca e la lingua latina. Sintetizzando, si hanno due teorie sull'origine della perifrasi, la poligenetica e la monogenetica. La prima teoria considera le perifrasi aspettuali come espressione di uno sviluppo interno alla lingua, la seconda le comprende nei fenomeni che a partire dal latino tardo si sono sviluppati nelle lingue romanze; al riguardo cf. L. AMENTA, Perifrasi aspettuali in greco e latino. Origini e grammaticalizzazioni (Milano, 2003, rist. 2007), pp. 12-13. H.St.J. THACKerAy, A Grammar of the Old Testament in Greek according to the Septuagint, Vol. I: Introduction, Orthography and Accidence (Cambridge, 1909, rist. Tel-Aviv 1970), p. 195 pone in rilievo l'influsso dell'ebraico sull'incremento d'uso della perifrasi verbale senza però farne

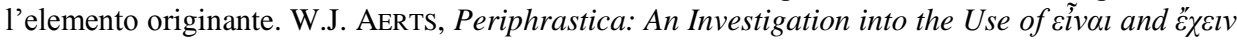
as Auxiliaries or Pseudo-Auxiliaries in Greek from Homer up to the Present Day (Amsterdam, 1965), pp. 61-62, 64 rivaluta l'influsso dei Lxx sull'affermazione della perifrasi. T. Evans, Verbal Syntax in the Greek Pentateuch: Natural Greek Usage and Hebrew Interference (Oxford, 2001), p. 256 ritiene fondata la teoria di Aerts ma con delle riserve. A differenza di quanto sostiene Aerts, la perifrasi progressiva e quella con il futuro non iniziano con i LXX. AMENTA, Perifrasi aspettuali, pp. 65-66 riprende la teoria di Aerts ma sostiene con Mandilaras (B.G. Mandarilas, Verb in the Greek Non-Literary Papyri [Athen, 1973], p.50) che la perifrasi è espressione anche di un processo endogeno alla lingua greca. Rimanendo nel perimetro della sola lingua greca, E. CoSERIU, "El Aspecto Verbal Perifrástico en Griego Antiguo", in Actas del III Congreso Español de Estudios Clásicos (Madrid, 1968), pp. 108-109 fa dipendere, a quanto sembra, la nascita/formulazione della perifrasi con presente e aoristo participio dalla mancanza nel greco di forme che esprimano una visione parziale dell'azione. 
gradualmente all'interno della lingua greca e l'apporto dei LXX e del $\mathrm{NT}^{4}$ abbia contribuito ad affermarla ulteriormente, bisogna stabilire quali siano le condizioni necessarie perché si abbia una perifrasi verbale, in altri termini quale sia il rapporto tra le due componenti (ausiliare e participio) e sotto quale aspetto cooperino a formarla. ${ }^{5} \mathrm{Si}$ impone naturalmente anche la questione della relazione tra le perifrasi e le forme sintetiche o monolettiche. ${ }^{6}$

4 Esempi di ristrutturazione del sistema verbale, i cui inizi sono rintracciabli nell'Attico e nei vari dialetti greci, appaiono già stabili nel periodo degli scritti neotestamentari e continuano ad operare nel periodo romano e primo bizantino. Al riguardo cf. R. BRownING, Medieval and Modern Greek (Cambridge, 1999), pp. 28-29. Sempre Browning registra che le costruzioni perifrastiche verbali sostituiscono vecchie forme "desistematizzate" e che la perifrasi composta da $\varepsilon i \mu i ́ / \tilde{\eta} v$ più presente participio non sostituisce il presente o l'imperfetto sintetici (32). Le osservazioni appaiono nel capitolo Greek in the Hellenistic World and the Roman Empire del volume citato.

5 PORTER, Verbal Aspect, pp. 449, 487 sostiene che si ha perifrasi solo con cỉ í per l'intrinseca genericità aspettuale del verbo. Tale genericità era stata affermata da C.J. RUJIGH, "A Review of Ch. H. Kahn, The Verb 'Be' in Ancient Greek", Lingua 48 (1979), p. 67: "eimi è il verbo meno marcato semanticamente della lingua". Evans, "Periphrastic Tense Forms", p. 223 riconosce che la nozione di genericità aspettuale di Porter contribuisce a definire la funzione dell'ausiliare nella perifrasi. Concorda con quest'ultimo, inoltre, nel sostenere che è il participio nella perifrasi a stabilire l'aspetto. In Verbal Syntax, p. 230 Evans afferma che in senso stretto si ha perifrasi, se c'è equivalenza con una forma temporale sintetica. La combinazione più produttiva è quella con

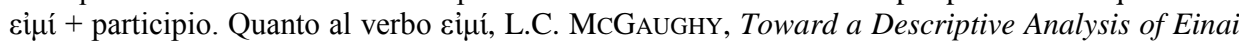
as a Linking Verb in the New Testament Greek, «SBL Dissertation Series» 6 (Montana, 1972), § 5.3 afferma: "its morphology distinguishes tense as time and mood, and it contributes to tense as aspect in periphrastic constructions".

Sul fenomeno della grammaticalizzazione del verbo finito che richiede la sua desemantizzazione e decategorizzazione cf. AmEnTA, Perifrasi verbali, pp. 20, 22, 25; tuttavia, secondo S.C. DiK, "Copula Auxiliarization: How and Why?", in M. HARRIS - P. RAMAT (ed.), Historical Development of Auxiliaries (Berlin, 1987), pp. 55-56, la copula nel processo di ausiliarizzazione non è sottoposta a desemantizzazione. Alcuni criteri per l'identificazione dell'ausialiarizzazione sono classificati da J. DE LA VILLA POLO, "La identificación de la auxiliaridad verbal en Griego", Cuadernos de Filología Clásica 22 (1989), pp. 197-198. L'autore tratta di verbi di movimento e altri verbi e non di cỉní, a cui dedica un solo accenno, rimandando al riguardo ad altri studiosi. Sono indici di ausiliarizzazione: il mutamento nell'argomentazione del verbo; restrizioni nella collocazione della negazione e di altri elementi dipendenti. Il primo criterio è valido nella costruzione con il participio, gli altri due con l'infinito. TH. MARKOPOULOS, The Future in Greek: From Ancient to Medieval (New York, 2009), pp. 11-13 rileva che delle ventidue proprietà che gli studiosi hanno individuato per definire l'uso di un verbo come ausiliare, le più condivise e convincenti sono tre: ausiliare e verbo devono avere il medesimo soggetto; il loro ordine è fisso; nessuno o pochi elementi devono interporsi.

6 M. Haspelmath, "Periphrasis", in G. Booij - Ch. Lehmann - J. Mugdan (ed.), Morphology: A Handbook on Inflection and Word Formation, Vol. 1, «Handbücher zur Sprach- und Kommunikationswissenschaft» (Berlin, 2000), p. 659 sottolinea che il processo di grammaticalizzazione delle perifrasi non opera per colmare una lacuna. Per lo studioso le perifrasi verbali e le forme sintetiche hanno una distribuzione complementare. Sulla stessa linea AMENTA, Perifrasi aspettuali, p. 132, secondo cui le perifrasi non sopperiscono a limiti del sistema verbale esistente ma tendono a esplicitare significati particolari. A tale proposito, soprattutto per la 
Evans osserva che nel periodo della koinè cỉ í più participio è la perifrasi verbale più frequente ${ }^{7}$. L'autore ha approfondito la costruzione della perifrasi verbale nei libri del Pentateuco. Riassumendo le conclusioni di Evans riguardanti questo capitolo del suo volume, scrivevo: "Nella determinazione dell'occorrenza di una perifrasi, concorrono inevitabilmente fattori soggettivi. Evans definisce la perifrasi una combinazione di un ausiliare più participio o infinito come quasi ("as near") equivalente (perifrasi sostitutiva) o supplenza (perifrasi suppletiva) di una forma sintetica. Negli ausiliari vanno considerati i tratti lessicali semantici e non quelli aspettuali al fine di stabilire la grammaticalizzazione della loro funzione. La teoria di Aerts, secondo cui i LXX hanno influito sull'uso della perifrasi sul greco del NT, è fondata ma non nei termini formulati dallo studioso, inoltre né la perifrasi progressiva né quella con il futuro prendono avvio dai $\mathrm{LXX}^{8}$. Per formulare conclusioni sulle perifrasi greche e l'influsso ebraico occorrono dati più esaustivi. Non è stato stabilito perché non si ha sempre perifrasi rispetto a una costruzione ebraica che potrebbe motivarla o rispetto a היה + participio. Gli studi, infine, non hanno prodotto dati sufficienti che permettano di studiare la frequenza della perifrasi nel Pentateuco rispetto a quella del greco extra biblico" ${ }^{9}$. Evans, interessato a studiare soprattutto il fenomeno della perifrasi nel Pentateuco in rapporto alla koinè e al testo ebraico tradotto, non discute sistematicamente le varianti ${ }^{10}$.

Di seguito sono elencate e discusse le varianti con cỉní più participio individuate nei testimoni della tradizione testuale del Pentateuco dei LXX $^{11}$. Saranno

funzione che la perifrasi può svolgere in un racconto, andrebbero rivalutate le osservazioni di J. GondA, “A Remark on 'Periphrastic' Construction in Greek”, Mnemosyne 4, ser. 12 (1959), pp. 99-102. Secondo questo autore, per fare un esempio, il passaggio dalla forma finita narrativa a quella perifrastica è riferibile alla necessità di sottolineare lo stato delle cose. Il contrasto tra le due forme, inoltre, emerge quando sono presenti entrambe in un medesimo contesto (101).

7 Evans, "Periphrastic", p. 112.

8 Per un quadro della teoria di Aerts sull'uso della perifrasi nel greco biblico cf. Evans, Periphrastica, pp. 60-62; 74-77.

9 Il virgolettato è ripreso da PIERRI, Perifrasi verbali, pp. 307-308, dove tra l'altro si aggiunge: "Queste considerazioni di Evans sono in parte condivisibili. L'autore, però, non discute le varianti (che possono offrire interessanti elementi sulla ricezione della perifrasi) e il tratto di quasi equivalenza delle perifrasi con le forme sintetiche: non dice in cosa si distinguono sostanzialmente le due forme. Attribuisce, infine, un'importanza decisiva al rapporto con l'ebraico. Con ciò non si vuol dire che un confronto con la lingua tradotta non possa essere illuminante, ma non è questa comparazione a produrre elementi discriminanti per stabilire se una costruzione sia perifrastica o no in greco, al più fornisce notizie sulla tecnica di traduzione e sull'interpretazione del traduttore. Stabilire che una perifrasi rispecchi un modello di traduzione è un fatto, un'altra è l'analisi in greco". Per le conclusioni a cui si fa riferimento cf. Evans, Verbal Syntax, 255-257.

10 Si può anticipare che le varianti sono per lo più piuttosto tardive.

11 Il testo critico adottato è quello dei volumi del Pentateuco nell'editio maior di Göttingen. Naturalmente saranno registrate anche le varianti relative al fenomeno inverso: forma sintetica in 
considerate anche le varianti con rívouar ma solo quelle concernenti il predicato nominale. La finalità è di osservare se vi siano tracce dell'affermazione della perifrasi nelle varianti, eventuali concentrazioni di frequenza insieme a sfumature d'uso, con particolare attenzione alla datazione dei testimoni dove appaiono. La disposizione delle attestazioni è strutturata per tempi. Un paragrafo è riservato anche alle varianti di costruzioni con predicato nominale (copula + aggettivo) rispetto a un verbo finito.

\section{Presente}

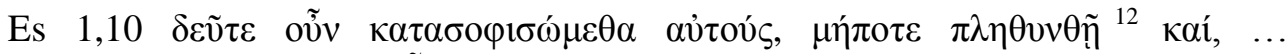

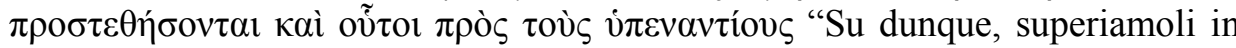
astuzia, perché non si moltiplichi e ... si aggiungano anche costoro agli avversari”.

Il gruppo di testimoni $x$ [71(XIII) - 527(XIV) - 619(XV)] ha $\pi \lambda \eta \theta 0 ́ v \omega v$ per $\pi \lambda \eta \theta v v \theta \tilde{\eta}$, un presente participio attivo in luogo di un aoristo congiuntivo passivo. Nella variante il verbo $\pi \lambda \eta \theta u ́ v \omega$ è intransitivo, la concordanza nel numero del

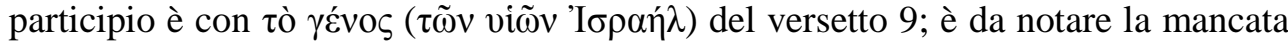
concordanza nel genere: se l'antecedente è $\tau$ ò $\gamma \varepsilon ́ v o \zeta$, il participio dovrebbe essere $\pi \lambda \eta \theta \tilde{v} v o v^{13}$. Nel contesto il participio $\pi \lambda \eta \theta v ́ v \omega v$ non sembra avere altra funzione se non di componente di una perifrasi verbale. Integrando l'ausiliare, la perifrasi può essere $(\mu \eta \dot{\pi} \pi \tau \varepsilon) \tilde{\tilde{n}} \pi \lambda \eta \theta \dot{v} v \omega v$, un presente congiuntivo equivalente alla forma sintetica $\pi \lambda \eta \theta \dot{v} v \eta$. Un'alternativa potrebbe essere $\gamma \varepsilon \dot{v} \eta \tau \alpha \Lambda$ $\pi \lambda \eta \theta \dot{v} v \omega v$. L'opposizione tra le due forme $\pi \lambda \eta \theta v v \theta \tilde{\eta}$ e $\tilde{\hat{\eta}} \pi \lambda \eta \theta v ́ v \omega v$ è aspettuale.

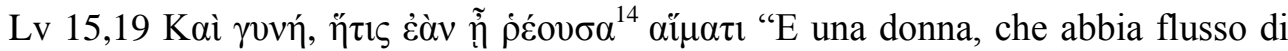
sangue".

luogo di cỉní più participio, perché una tale variante è prova a favore dell'uso perifrastico del participio.

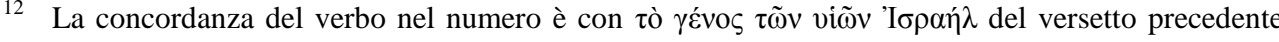
(9). Il passivo ha valore mediale. Per un'altra interpretazione dell'uso del singolare cf. J.W. Wevers, Notes on the Greek Text of Exodus, «Septuagint and Cognate Studies. Society of Biblical Literature» 30 (Atlanta, 1990), p. 4: "Presumably Exod uses the singular $\pi \lambda \eta \theta v v \theta \tilde{\eta}$ intentionally; individuals do not becomes numerous, but a people can, and as individuals they can join themselves to the ranks of the enemy, engage in war and leave the country".

13 Per la mancata concordanza nel genere cf. BRowning, Medieval and Modern Greek, p. 64, dove si dice che il participio subisce una graduale e inarrestabile desistematizzazione. Non solo si ha confusione tra i tempi del participio attivo, tra i generi e i casi, ma si incontrano participi coordinati con verbi finiti. Queste osservazioni si trovano nel capitolo The Greek language in the later middle ages (1100 - 1453) del volume citato.

14 Evans, Verbal Syntax, p. 234, analizza la perifrasi come presente congiuntivo e ricorda il parallelo con 15,25; cataloga la perifrasi in esame come sostitutiva (250). AERTs, Periphrastica, 3 
Il gruppo di testimoni $b$ [19(XII) -108(XIII) -118(XI-XII) - 314(XIII) -537(XIII)] ha $\rho \dot{q} \eta$

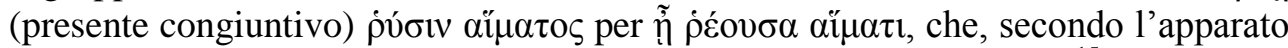

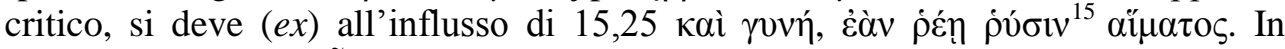

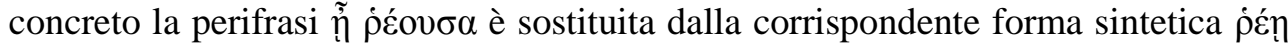

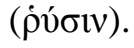

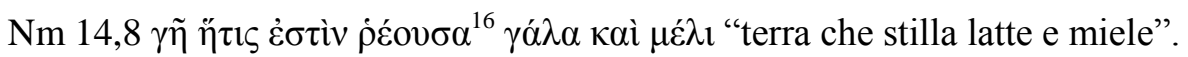

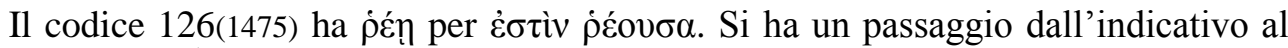
congiuntivo ${ }^{17}$.

\section{Imperfetto}

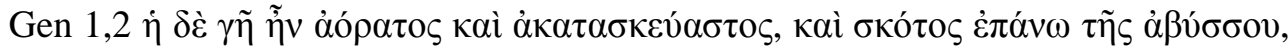

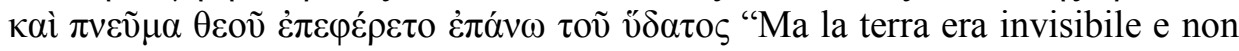
preparata, e la tenebra stava sull'abisso, e lo spirito di Dio si portava sull'acqua".

Siccome le varianti sono tratte dai 'Tre', per ragioni cronologiche riprendiamo in

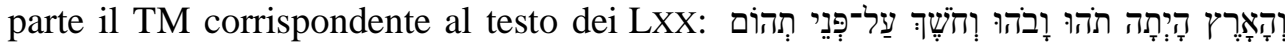

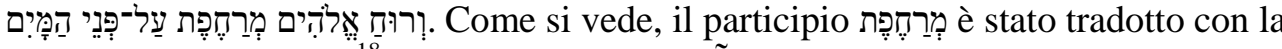
forma finita $\dot{\varepsilon} \pi \varepsilon \varphi \varepsilon \dot{\varepsilon} \rho \tau \mathrm{o}^{18}$ coordinata con $\kappa \alpha i ́$ a $\tilde{\eta} \nu$.

distingue tre tipi di perifrasi verbali. È sostitutiva, quando sostituisce una forma monolettica senza sostanziali differenze; è suppletiva, quando sostituisce una forma monolettica non esistente o non più usata; è espressiva, nel caso abbia un particolare scopo. Evans, Verbal Syntax, 221-222, nel commentare la tripartizione proposta da Aerts, ritiene il terzo tipo marcato da eccessiva soggettività e perciò non l'assume. J.W. WEVERS, Notes on the Greek text of Leviticus, «Septuagint and Cognate Studies. Society of Biblical Literature» 44 (Atlanta, 1997), p. 232 rileva,

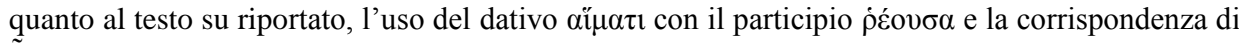
רิ

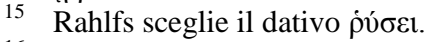

16 La perifrasi è registrata come presente indicativo in Evans, Verbal Syntax, p. 234; la perifrasi è sostitutiva (252). Cf. inoltre F.C. CONYBEARE - G. STOCK, A Grammar of Septuagint Greek (Boston, 1905, rist. Zondervan 1981), p. 68.

17 La forma $\rho \dot{\varepsilon ́ n}$ nel contesto è terza persona singolare attiva del presente congiuntivo di $\rho \dot{\varepsilon ́} \omega$. Il congiuntivo ha una sfumatura finale. Per questa funzione del modo nelle proposizioni relative cf. F. Blass - A. DebrunNer - F. ReHKoph [BDR], Grammatica del Greco del Nuovo Testamento, «Supplementi al "Grande Lessico del NT"» 3 (Brescia, 1982), § 379. Si veda anche A.N. JANNARIS, An Historical Greek Grammar Chiefly of the Attic Dialect as written and spoken from Classical Antiquity down to the Present Time. Founded upon the Ancient Texts, Inscriptions, Papyri and Present Popular Greek (Hildesheim: Olms, 1987), § 2022.

18 J.W. Wevers, Notes on the Greek Text of Genesis, «Septuagint and Cognate Studies. Society of Biblical Literature» 35 (Atlanta, 1993), p. 3 vede in questa corrispondenza l'intenzione di esprimere il "continuous character" del participio. 


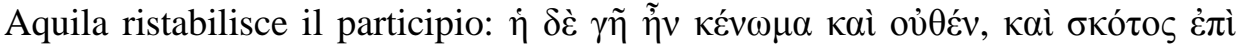

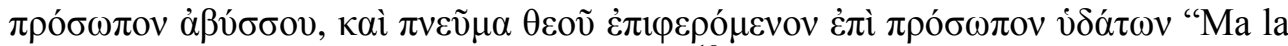
terra era uno spazio vuoto e non c'era nulla ${ }^{19}$, e (la) tenebra stava sulla superficie dell'abisso, e lo spirito di Dio si portava sulla superficie delle acque ${ }^{20,}$. Appare evidente che $\tilde{\eta} v$ unisce come copula $\gamma \tilde{\eta}$ con $\kappa \varepsilon ́ v \omega \mu \alpha$ e oủ $\theta \dot{\varepsilon} v$, e funge da predicato

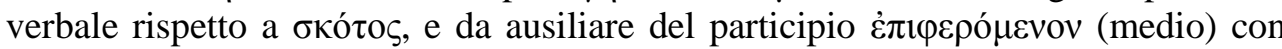

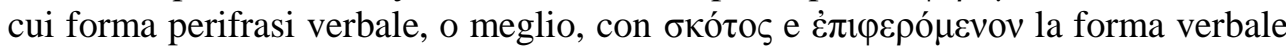
è omessa. Teodozione e Simmaco hanno la stessa costruzione e il medesimo participio, ma Simmaco ha $\dot{\varepsilon} \gamma \varepsilon \dot{\varepsilon} v \varepsilon \tau$ in luogo di $\tilde{\eta} v^{21}$. Si può supporre anche una

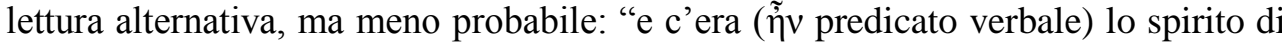

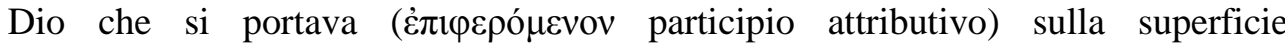
dell'acqua".

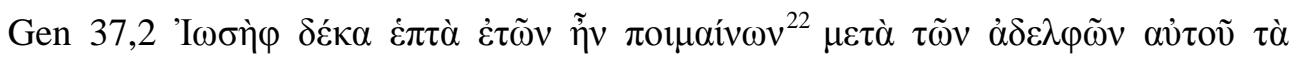
$\pi \rho o ́ \beta \alpha \tau \alpha$ ôv vé́o "Giuseppe aveva diciassette anni; stava pascolando con i suoi fratelli le pecore, quando era giovane".

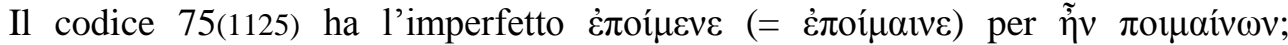

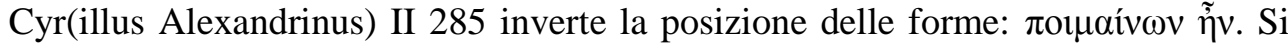
può avere, dunque, anche il fenomeno inverso, si passa dalla perifrasi alla forma sintetica. Inoltre la teoria secondo cui la perifrasi verbale richieda la successione 'ausiliare - participio' non sembra essere così rigida, stando alla scelta di Cirillo Alessandrino (370 - 440). È evidente la corrispondenza aspettuale tra le lezioni.

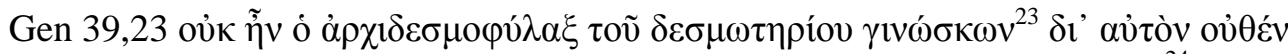
"Il capo carceriere del carcere non conosceva (non si sforzava di sapere ${ }^{24}$ ) nulla grazie a lui (Giuseppe)".

19 È probabile che Aquila intenda oủyév come sostantivato: "un vuoto, uno spazio". La traduzione proposta presenta una variazione stilistica dove la forma ĩ $v$ è resa come predicato verbale.

20 Evans non accenna al testo di Aquila.

21 L'analisi secondo Evans, Verbal Syntax, p. 227 non cambia, si tratta di imperfetto indicativo, secondo il principio condiviso dagli studiosi, per cui è il participio a determinare l'aspetto della perifrasi. L'autore, in realtà, è più sfumato: il participio "provide(s) the dominant aspectual contribution to the periphrasis". Evans precedentemente scrive: "Even cijui overlaps partially the semantic range of $\gamma^{\prime} v o \mu \alpha 1$, and $\dot{\gamma} \gamma \varepsilon v o ́ \mu \eta v$ in the sense "was' can arguably function as its aorist" (223).

22 Per la perifrasi cf. Evans, Verbal Syntax, p. 237; definisce la perifrasi come sostitutiva (250). Registrano la perifrasi anche CONYBEARE - STOCK, A Grammar, p. 69.

23 Per la perifrasi cf. Evans, Verbal Syntax, p. 237; 1'autore definisce la perifrasi come sostitutiva (252).

24 Il contesto (Gen 39,21-23) dice espressamente che Dio assiste Giuseppe e che il capo carceriere si fida del tutto di quest'ultimo. 


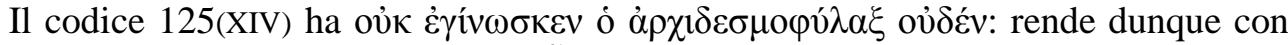

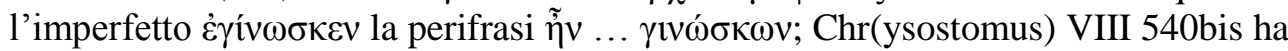

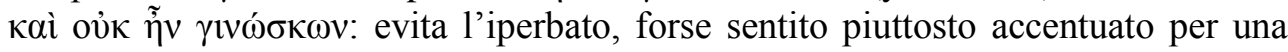
perifrasi. La scelta di Giovanni Crisostomo (344/354 - 407) è un esempio a sfavore della teoria di Porter, secondo cui l'inserzione di elementi tra ausiliare e participio rende incerta l'analisi della costruzione come perifrasi verbale ${ }^{25}$ : Crisostomo pone di seguito i due membri ma, anche se distanti nel testo originale, li riconosce come formanti una perifrasi.

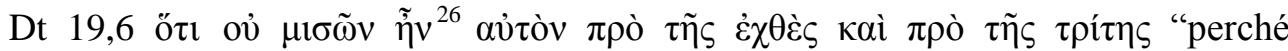
(l'omicida involontario, v. 4) non lo odiava prima di ieri e dell'altro ieri".

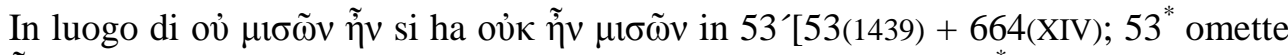
$\tilde{\eta} v]$; l'omissione dell'ausiliare si registra anche nei codici $610^{*}$ (XIV) 54(XIII-XIV)$75^{*}(1125)$. Nei codici 53(1439) - 664(XIV) si ristabilisce l'ordine più consueto dei membri della perifrasi: ausiliare - participio.

\section{Futuro}

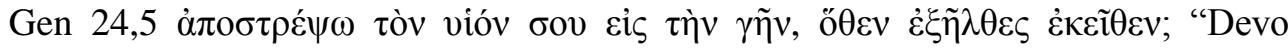
portare indietro tuo figlio nella terra da dove sei uscito?".

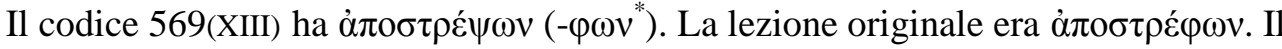
futuro indicativo perifrastico con ausiliare sỉ verbo finito e il participio presente. L'adozione del futuro participio è segno della possibilità di una perifrasi verbale formata da entrambe le componenti al futuro o con l'ausiliare al presente? Se si scarta la perifrasi, l'unica alternativa, considerata anche l'altezza cronologica del codice 569 , è l'uso indipendente del participio ${ }^{27}$.

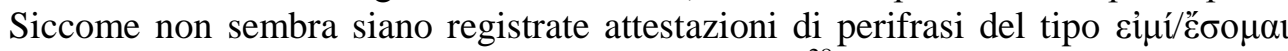
più futuro participio, si può sostenere il secondo uso ${ }^{28}$.

25 PoRTER, Verbal Aspect, pp. 492-493. Per l'autore si ha perifrasi verbale, se c'è un verbo ausiliare con aspettualità generica e se il participio concorda con il suo referente. Altra condizione perché vi sia perifrasi è l'adiacenza dei due membri. Eventuali elementi aggiunti devono essere connessi al participio, se, invece, sono in relazione al verbo finito, provano l'indipendenza di quest'ultimo (che non è perciò ausiliare). L'inserzione di un sintagma locativo o temporale tra i due membri crea incertezza nell'analisi. La medesima teoria è ribadita in S.E. PORTER, Idioms of the Greek New Testament, «Biblical Languages: Greek» 2 (Sheffield, 1992), pp. 45-46. Per una critica alla posizione di Porter cf. Evans, "Periphrastic", p. 233.

26 Evans, Verbal Syntax, p. 238: imperfetto indicativo; p. 252: perifrasi sostitutiva.

27 Cf. sopra nota 13.

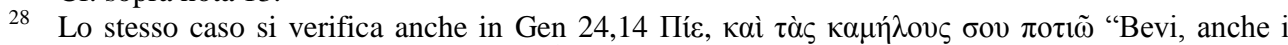

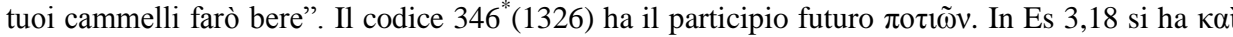




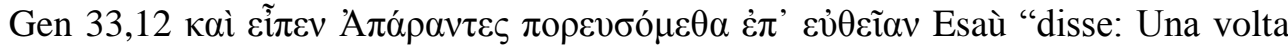
partiti, andremo diritto".

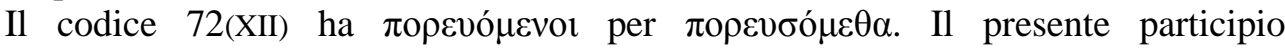

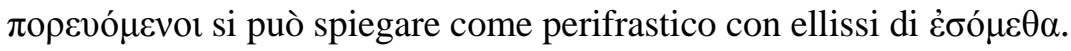

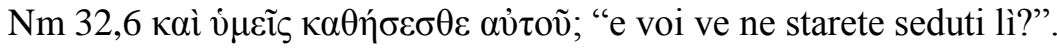

Il codice 799(1280) ha $\check{\varepsilon} \sigma \varepsilon \sigma \theta \varepsilon \kappa \alpha \theta \eta \dot{\mu} \mu \varepsilon v o r$ per $\kappa \alpha \theta \eta \dot{\sigma} \sigma \varepsilon \sigma \theta \varepsilon$. L’equivalenza aspettuale è rispettata.

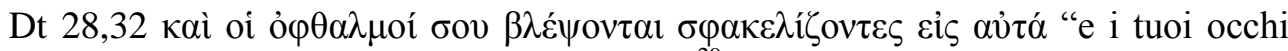
guarderanno verso di loro consumandosi" 29 .

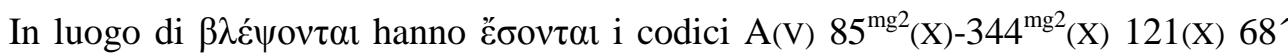

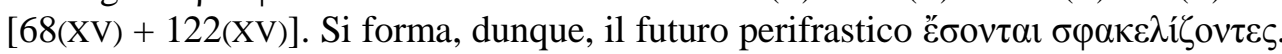
Omettono siç i codici 848(vid) $12168^{\prime}$. Con la perifrasi il senso è "e i tuoi occhi si consumeranno riguardo a loro".

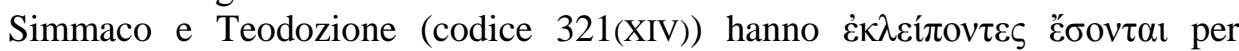
$\sigma \varphi \alpha \kappa \varepsilon \lambda i \zeta o v \tau \varepsilon \varsigma$, per cui si ha: “... guarderanno (e) verranno meno riguardo a loro".

\section{Futuro perfetto}

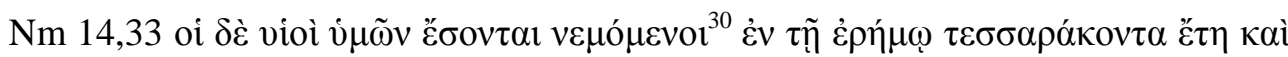

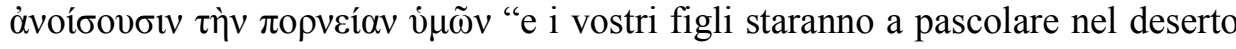
quarant'anni e prenderanno su di sé la vostra prostituzione".

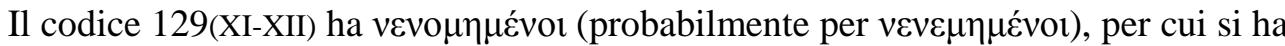
futuro perfetto. La variante dimostra che il participio perfetto è sentito come forma verbale e non aggettivale.

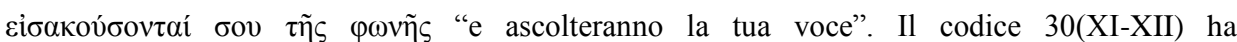

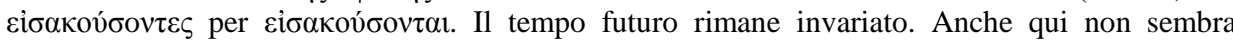

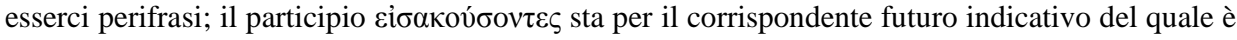
variante. Ipotizzare che il participio sia il risultato della lettura del dittongo al come $\varepsilon$ e dell'aggiunta del sigma finale $(-\varepsilon \varsigma)$, per influsso del pronome бoṽ successivo, è un'ipotesi poco sostenibile.

29 "Occhi", in questo contesto, sta per animo. Nel passo in esame il senso di $\sigma \varphi \alpha \kappa \varepsilon \lambda i \zeta \omega$ è traslato e non fa riferimento alla putrefazione concreta. Certamente più accettabili le traduzioni di L.C.L. BRENTON, The Septuagint with Apocrypha: Greek and English, I (London, 1851, rist. Grand Rapids, 1980), p. 269: "and thine eyes wasting away shall look for them”, e di L. MORTARI (ed.), La Bibbia dei LXX: 1. Il Pentateuco, Roma 1999, 855 "e i tuoi occhi si seccheranno nel guardare...”, rispetto a quella di A. PiETERSMA - B.G. Wright (ed.), A New English Translation of the Septuagint, (New York, 2007), p. 167: "and your eyes, being purulent, shall look for them".

30 Evans, Verbal Syntax, p. 241: futuro indicativo; p. 250: sostitutiva. Esempio registrato erroneamente da CONYBEARE - StOcK, A Grammar, p. 68 come Dt 14,33. 


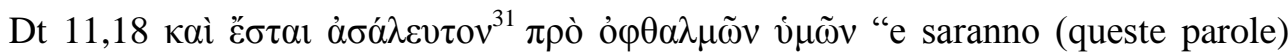
fisse davanti ai vostri occhi”.

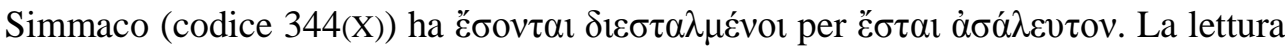
di Simmaco deve essere "e staranno (ben) distinte davanti ai vostri occhi".

\section{Aoristo $^{32}$}

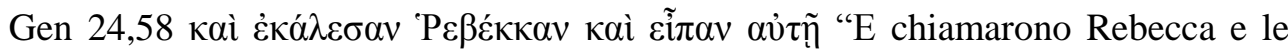
dissero".

Il codice $799(1280)$ ha il participio $\kappa \alpha \lambda \varepsilon ́ \sigma \alpha \nu \tau \varepsilon \varsigma$ per l'indicativo $\varepsilon \kappa \alpha ́ \lambda \varepsilon \sigma \alpha \nu$ e non elimina il secondo кaí. Si può pensare a un mancato participio congiunto: "E,

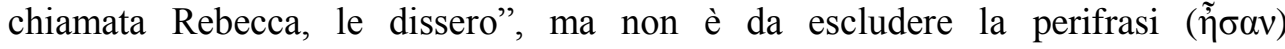
$\kappa \alpha \lambda \varepsilon ́ \sigma \alpha \nu \tau \varepsilon \zeta$, che probabilmente è intesa a esprimere l'anteriorità. Tenuto conto, tuttavia, della data del codice, si può pensare anche ad un uso 'improprio' del participio $^{33}$.

31 Wevers, nell'edizione critica, sceglie $\dot{\alpha} \sigma \alpha ́ \lambda \varepsilon v \tau \alpha$, ma la lezione $\dot{\alpha} \sigma \alpha ́ \lambda \varepsilon v \tau o v$ è attestata, tra gli altri codici, in A B F M V.

32 AERTs, Periphrastica, p. 27 osserva che in linea di principo la costruzione Eĩval + aoristo participio differisce nell'aspetto dal presente e dal perfetto: esprime azione e non stasi o situazione stabile. L'autore successivamente (35) rileva che ad uno sguardo generale le costruzioni con aoristo participio e eĩval ( $\left.\gamma^{\prime} \gamma v \varepsilon \sigma \theta \alpha 1\right)$ in molti casi sono perifrastiche. L'uso si può spesso ricondurre a circostanze particolari. L'equivalenza con il piuccheperfetto latino postulata da Björck e altri autori non può essere assunta senza verifica. La perifrasi con aoristo ha carattere aspettuale e non ricopre altre funzioni o sostituisce altre forme aspettuali. Ad ogni modo per Aerts (76-77) nei LXX la perifrasi عĩvol + aoristo participio non è attestata, ha valore di piuccheperfetto e si afferma nel primo secolo. In BDR, § 355 circa la perifrasi cĩval + aoristo participio si legge che, usata nel greco classico "per dare risalto all'idea verbale, serve più tardi ad esprimere il

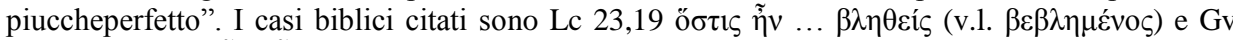

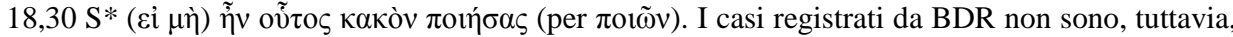

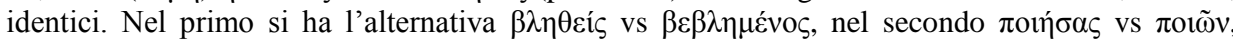
dove, a rigore, si ha un'opposizione tra piuccheperfetto e imperfetto perifrastici. In H. STEPHANUS (ed.), Thesaurus graecae linguae, IV (Graz, 1954), coll. 258-259 sub voce eỉní, si registra la

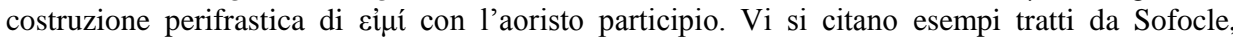
Eschilo, Erodoto e Senofonte. Nei passi cỉuí è al presente (due indicativi e un ottativo), futuro (quattro indicativi e un infinito) e imperfetto. La perifrasi ausiliare - aoristo participio, dunque, è attestata anche nel greco più antico.

Nonostante gli autori (vedi BDR) accettino l'equivalenza síní + aoristo participio = piuccheperfetto, si preferisce tenere distinte le costruzioni con l'aoristo participio da quelle con perfetto participio (ossia imperfetto di $\varepsilon i \mu$ í + perfetto participio). Tale equivalenza riguarderebbe solo l'indicativo, ma, stando al Thesaurus, le combinazioni tra verbo finito e aoristo participio possono riguardare anche altri tempi e modi (vedi l'ottativo).

33 JANNARIS, An Historical Greek Grammar nel § 2167 osserva che, negli autori del periodo postclassico e neollenico, l'uso del participio nella varietà espressiva dell'Attico è confinato a un 


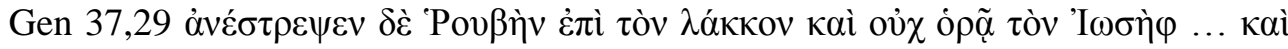

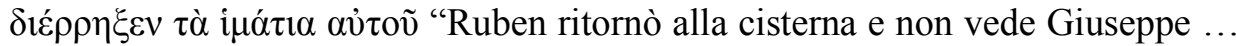
e si stracciò le vesti”.

Il codice 458(XII) ha غ̇ंı

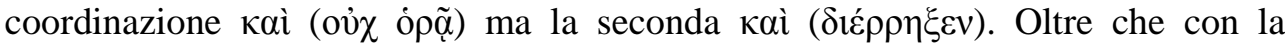
perifrasi verbale, in questo caso, forse, l'adozione del participio si può spiegare anche come un tentativo mal riuscito di cambiare la successione di indicativi aoristi con un participio congiunto temporale. Si tenga presente, tuttavia, quanto affermato nel paragrafo precedente.

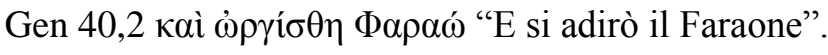

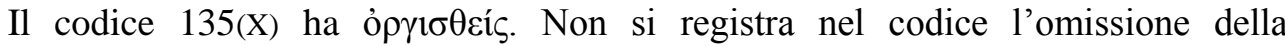

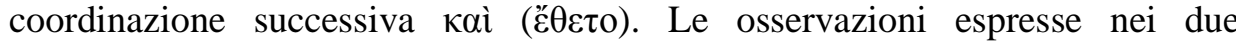
paragrafi precedenti valgono anche in questo caso.

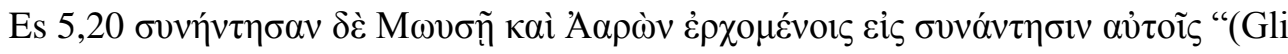
scribi degli israeliti) Andarono incontro a Mosè e ad Aronne che venivano per incontrarli”.

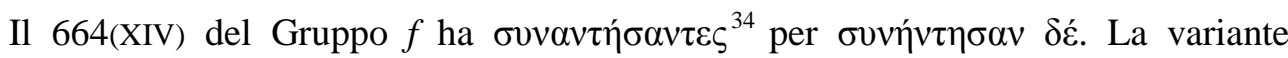
elimina la coordinazione. Si ha quindi: "Andati incontro a Mosè e ad Aronne..." ".

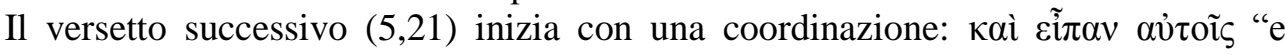
dissero loro".

Ipotizzare un uso perifrastico del participio non è escluso, come non è da escludere che si possa analizzare come congiunto. Il participio congiunto, in genere, è preceduto o seguito dalla congiunzione (se c'è) che coordina (in genere) il verbo della reggente ${ }^{35}$. Il $\kappa \alpha i$ del versetto 21 è piuttosto distante dal participio ed è ovviamente prepositivo rispetto a $\varepsilon \tilde{i} \pi \alpha v$ e forse potrebbe giustificare l'eliminazione della congiunzione $\delta \varepsilon ́$. Il risultato del cursus del periodo, però, non è dei più riusciti. La perifrasi ( $\tilde{\eta} \sigma \alpha \nu \sigma v v \alpha v \tau \eta \dot{\sigma} \sigma \alpha v \tau \varepsilon \varsigma$ ), anche se asindetica, ristabilisce una certa fluidità sintattica tra i due versetti.

uso scolastico, tanto che alcuni autori, pur ben preparati, commettevano errori nell'impiego del participio circostanziale. Nel paragrafo successivo (2168) ricorda che il participio circostanziale è diventato estraneo alla lingua parlata dal 300-600 d.C., se non prima, e che ne sono segni: a) l'uso e la costruzione erronea, in particolare con il genitivo e nominativo assoluti; b) l'identificazione con il verbo finito o l'infinito e il conseguente errore d'uso come verbo indipendente finito o coordinato con $\kappa \alpha$ í. Cf. inoltre sopra la nota 13.

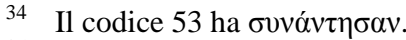

35 I modelli principali sono: $\kappa \alpha i ́$ + participio oppure participio + coordinante pospositiva. 


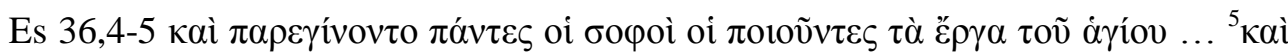

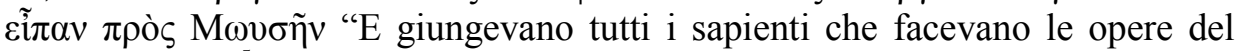
luogo santo ... 5 e dissero a Mosè".

Diversi manoscritti (reliqui) hanno $\pi \alpha \rho \alpha \gamma \varepsilon v o ́ \mu \varepsilon v o r$ (aoristo participio) per $\pi \alpha \rho \varepsilon \gamma$ ívovto (imperfetto indicativo) ${ }^{36}$. La variazione di tempo sembra rispondere a prima vista più all'introduzione di un participio congiunto che all'adozione di una perifrasi verbale. La coordinazione del participio con $\varepsilon \tilde{i} \pi \alpha v$ orienta, tuttavia, a un suo uso perifrastico o come verbo finito.

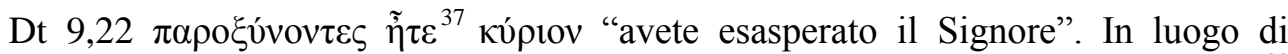

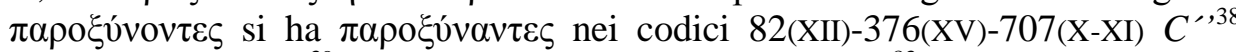
$b(19-108-118-314-537)^{39} 56^{\prime}[56(1096)+246(1195)] 458$ (XII) $z^{-83}(18-68-120-122-128-630-$ $669)^{40} 59(\mathrm{XV}) 319$ (1021) 646(XII).

In questo caso non si possono chiamare in causa elementi contrari o concorrenti all'uso della perifrasi con il participio aoristo ${ }^{41}$. La perifrasi è evidente. Si ha, dunque, un'opposizione aspettuale tra un imperfetto e un aoristo perifrastici.

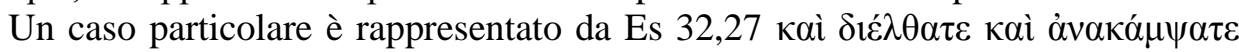

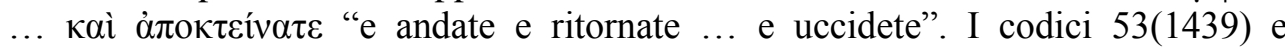

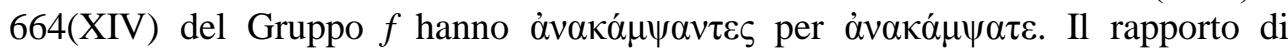
coordinazione non subisce modifiche: il terzo kaí non viene eliminato, per cui non

36 A proposito del participio WeVERs, Notes Exodus, p. 594 osserva: “The majority A F M tradition has the aorist participle $\pi \alpha \rho \alpha \gamma \varepsilon v o ́ \mu \varepsilon v o 1$ which is puzzling. Il may have originated under the

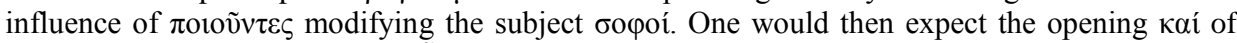
v.5 to be omitted as well, since eĩ $\pi \alpha v$ then becomes the main verb, but only a few mss attest that omission".

37 Evans, Verbal Syntax, 238 definisce la perifrasi imperfetto indicativo; 251: sostitutiva. Benché non sia strettamente attinente alla ricerca in corso, va rilevato che nel testo critico Wevers opta per la lezione $\pi \alpha \rho \circ \xi_{v} v o v \tau \varepsilon \varsigma \tilde{\eta} \tau \varepsilon$ кúptov, ma l'autore in Notes on the Greek text of Deuteronomy, «Septuagint and Cognate Studies. Society of Biblical Literature» 39 (Atlanta, 1995), p. 169 corregge il testo (rivalutando il numero delle attestazioni) con l'aggiunta dell'apposizione:

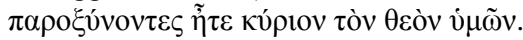

38 La sigla su riportata comprende ben ventitre codici, dei quali solo tre non sono manoscritti catenari: 16131 (dello stesso sottogruppo) e 46. La datazione di tutti e ventitre va dal X al XV secolo. Sulla letteratura catenaria e sulla sua importanza cf. N. FERNÁNDEZ MARCOS, La Bibbia dei Settanta. Introduzione alle versioni greche della Bibbia (Brescia, 2000), pp. 281-293 (con bibliografia).

39 I manoscritti del Gruppo $b$ datano tra l'XI e il XIII secolo.

40 I manoscritti del Gruppo $z$ datano tra l'XI e il XV secolo; il codice 83, che non ha la lezione in esame, risale al XVI secolo.

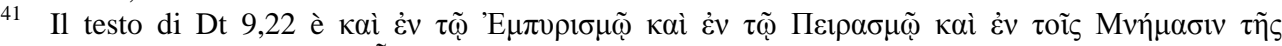

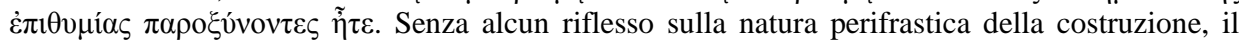
codice 319 premette кaí - in questo caso con funzione di particella additiva ("anche") - a $\pi \alpha \rho \circ \xi \tilde{v} \alpha \nu \tau \varepsilon \varsigma \tilde{\eta} \tau \varepsilon$. 


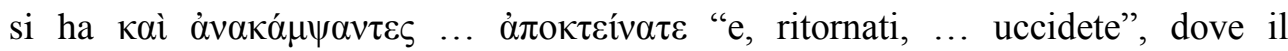
participio sarebbe con più evidenza congiunto (temporale) al soggetto di $\dot{\alpha} \pi$ $\dot{\alpha} v \alpha \kappa \alpha ́ \mu \psi \alpha \nu \tau \varepsilon \zeta$, anche se è proponibile pensare a un uso imperativale del participio $^{42}$.

\section{Perfetto}

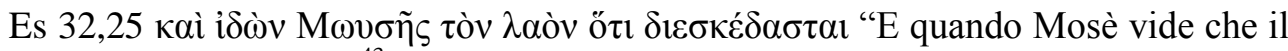
popolo si era disperso" $"$.

42 L'uso del participio-imperativo non è da tutti accettato allo stesso modo. Si esprime parere favorevole in BDR, $\S 468 b$ e nota 5 senza alcun riferimento alla perifrasi. Circa l'integrazione di

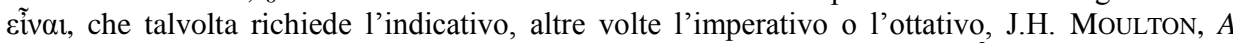
Grammar of New Testament Greek, Vol. I: Prolegomena (Edinburgh, 1908', rist. 1978), p. 182 osserva: "The fact is, when we speak of a part of Ẽ̃val being 'understood', we are really using inexact language". L'integrazione di ह̌ $\sigma \tau \varepsilon$ con i participi attestati in Rm 12,9s.16; Col 3,16; 2Cor 9,11.13; 8,24; Ef 4,2 (l'autore rimanda anche ad altri testi elencati da Regard) non è obbligatoria secondo J.H. Moulton - N. TuRner, A grammar of New Testament Greek, Vol. III: Syntax

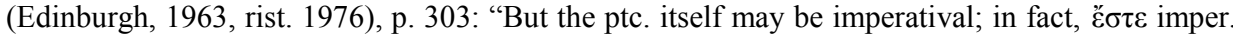
does not occur at all in NT"' Il medesimo concetto è ribadito successivamente (308). Ancora sul participo imperativale in MOULtON-TURNER III, 343 si legge: "The imperatival ptc. is a well known phenomenon in Peter and Paul, and it is common in the Koine. However, as है $\sigma \tau \varepsilon$ (imperat.) never occurs in NT, we must presume that it is understood as a copula with all these ptcs., which therefore do not constitute an anacolouthon". Gli autori notano che in 2Cor 8,4 il participio $\dot{\varepsilon} v \delta \varepsilon ı \kappa v v ́ \mu \varepsilon v o l$ ha come variante l'imperativo $\dot{\varepsilon} v \delta \varepsilon \dot{\xi} \xi \alpha \sigma \theta \varepsilon$.

Vanno fatte alcune osservazioni sull'ultima analisi di Moulton-Turner. E' molto probabile che i due autori parlino di copula in luogo di ausiliare. Nell'alternativa con la variante, poi, si passa

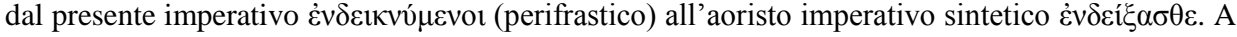
una verifica dei tempi dei participi nelle attestazioni citate dagli autori, emerge che la perifrasi all'imperativo predilige il presente participio. Circa la discussione sulla necessità o meno di integrare l'ausiliare, inoltre, non si vede perché non ipotizzare la sua ellissi. A parte quanto si è detto nella nota 32 sui tempi e modi dell'ausilare nelle attestazioni di perifrasi con aoristo participio in Sofocle, Eschilo, Erodoto e Senofonte, solleva più di qualche perplessità che questo tipo di perifrasi possa alla fine esprimersi solo all'indicativo con la teorizzata equivalenza con il piuccheperfetto.

Sempre in BDR, $\S 468 b$ nota 5 si registrano come imperativali anche gli aoristi participi medi $\dot{\alpha} \pi \varepsilon \kappa \delta v \sigma \alpha ́ \mu \varepsilon v o r$ (Col 3,9) e $\dot{\varepsilon} v \delta v \sigma \alpha ́ \mu \varepsilon v 01$ (Col 3,10), che sono con più probabilità participi congiunti temporali. Sull'uso del participio come modo finito nei papiri tolemaici esprime riserve E. MAYSER, Grammatik der griechischen Papyri aus der Ptolemäerzeit, II,1 (Berlin, 1970), pp. 340-341. Stando all'autore, se pure alcuni casi isolati di uso del participio come verbo finito sono possibili nel Nuovo Testamento e nella letteratura successiva, non emergono prove stringenti per un uso analogo nei papiri; con rimando alla nota 3 della pagina 196 ricorda di aver provato l'infondatezza dell'uso imperativale del participio nei papiri (340). Lo stesso discorso vale anche per gli altri modi finiti. Per Mayser detti participi sono "strafalcioni" o "anacoluti", oppure "treten lautliche bzw. syntaktische Erklärungen und Richtingstellungen in Kraft; manchmal ist Text und Zusammenhang unsicher" (341).

43 Evans, Verbal Syntax, p. 159 parla del sistema del perfetto ma non della variante. 


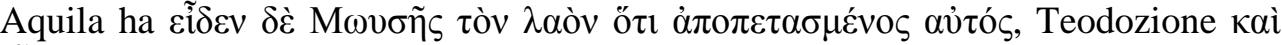

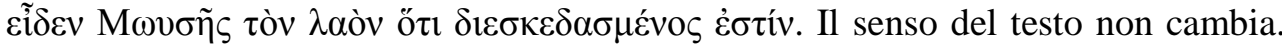
Cambia invece la costruzione del perfetto: a $\delta 1 \varepsilon \sigma \kappa \varepsilon ́ \delta \alpha \sigma \tau \alpha$ l si oppongono

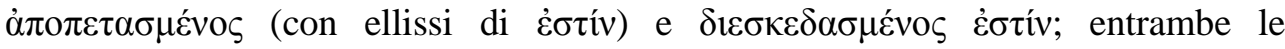
costruzioni sono perifrasi verbali.

Nm $5,14 \alpha$ ... ma lei non sia contaminata".

Invece di $\mu \varepsilon \mu i^{\prime} \alpha v \tau \alpha$ hanno $\tilde{\eta}$ $\mu \varepsilon \mu 1 \alpha \mu \varepsilon^{\prime} \eta 16^{\mathrm{c}}$ (XI)-46(XIII-XIV)-77(XIII-XIV) ${ }^{45}-500^{\prime}$ [500(XI-XII)-739(X)]-529'529(XIII)-616(XI)].

Anche se con una leggera differenza ortografica $(\mu \varepsilon \mu 1 \alpha \mu$. vs $\mu \varepsilon \mu 1 \alpha \mu \mu$.), i testimoni citati uniformano l'indicativo $(\mu \varepsilon \mu i ́ \alpha v \tau \alpha \mathrm{l})$ precedente al congiuntivo perifrastico successivo. È un intervento stilistico dovuto alla congiunzione subordinante $\dot{\varepsilon} \grave{\alpha} v$

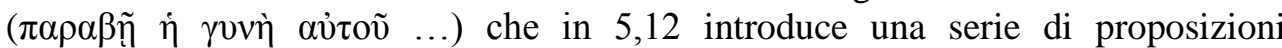
ipotetiche.

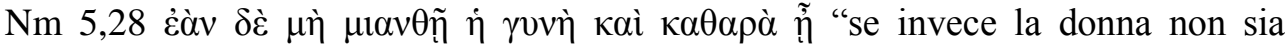
contaminata e sia pura".

Invece dell'aoristo passivo $\mu 1 \alpha v \theta \tilde{\eta}$ hanno il perfetto passivo $\tilde{\eta} \mu \varepsilon \mu 1 \alpha \mu \mu \varepsilon ́ v \eta$ i codici $68^{\prime}\left[68(\mathrm{XV})+122(\mathrm{XV})^{46}\right]-120^{\prime}[120(\mathrm{XI})+407$ (fine $\left.\mathrm{IX})\right]$. La perifrasi si può giustificare per ragioni aspettuali ${ }^{47}$.

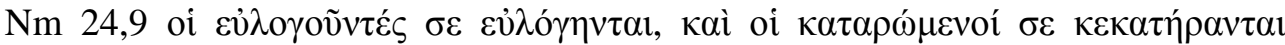
"coloro che ti benedicono sono benedetti e coloro che ti maledicono sono maledetti".

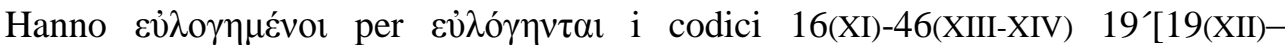
108(XIII)] $d$ [44(XV)-106(XIV)-107(1334)-125(XIV)-610(XIV)] $n \quad$ [54(XIII-XIV)75(1125)-127(X)-458(XII)-767(XIII-XIV)] $130^{\mathrm{mg}}$ (XII-XIII)-321 ${ }^{\text {mg }}$ [321(XIV)-346(1326)] $344^{\mathrm{mg}} t$ [74(tardo XIII)-76(XIII)-84(X-XI)-134(XI)-370(XI)] 126(1475).

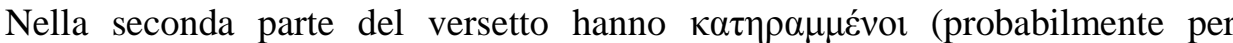

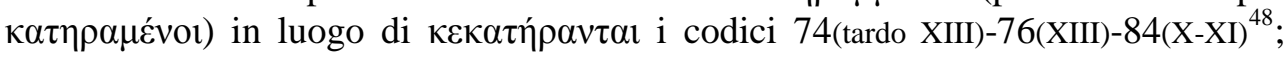

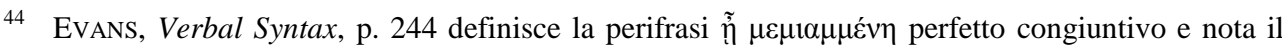
contrasto con il precedente perfetto; p. 254: suppletiva. La medesima forma è attestata anche in $\mathrm{Nm}$ 5,13.27.

45 Il codice 77 ha $-\alpha \sigma \mu \varepsilon \dot{v \eta}$.

46 Il codice 122 ha $-\alpha \mu \varepsilon ́ v \eta$.

47 J.W. Wevers, Notes on the Greek text of Numbers, «Septuagint and Cognate Studies. Society of Biblical Literature» 46 (Atlanta, 1998), p. 92 nel commentare la variante $\mu \varepsilon \mu$ í $\alpha v \tau \alpha$ l (indicativo) rispetto all'aoristo $\mu \iota v \theta$ ñ osserva: "This seems to be an exegetical attempt to show that the woman had been and still was undefiled, ... it is rather a suspected case of adultery, and the aorist as default tense in the correct form". 


\author{
106(XIV)-125'107(1334) + 125(XIV)] 134(XI)-370(VIII) ${ }^{49}$; il codice 767(XIII-XIV) ha

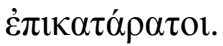

Le varianti possono essere dovute alla ricerca di simmetria tra participi: (oi)

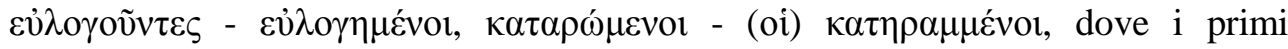
participi (con articolo) sono sostantivati, i secondi sono perifrastici (predicati

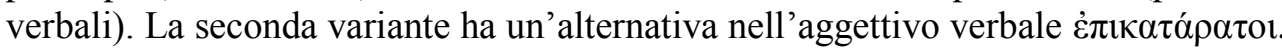
L'aggettivo non dimostra che i participi sono aggettivati e che, in ultima analisi,

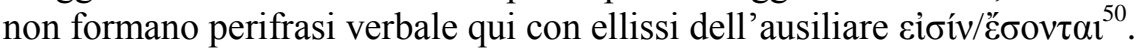

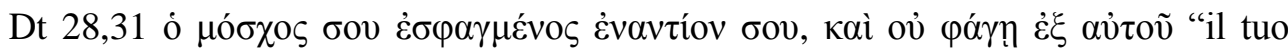
vitello è (stato) sgozzato davanti a te, ma tu non ne mangerai", ma anche "sarà sgozzato" ${ }^{\text {"51. }}$.

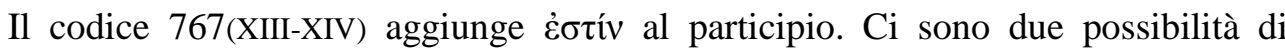
interpretare la variante. La prima, più probabile e già espressa nella traduzione

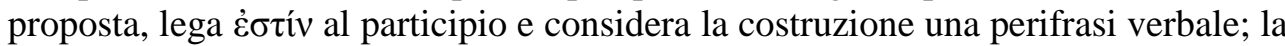
seconda riferisce il verbo alla preposizione $\dot{\varepsilon} v \alpha v \tau i ́ o v$, per cui si ha: "il vitello una volta sgozzato (participio congiunto) è (predicato verbale) davanti a te, ma tu non ne mangerai" ${ }^{\prime 52}$.

\title{
Piuccheperfetto
}

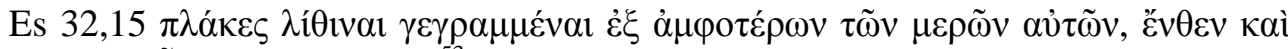

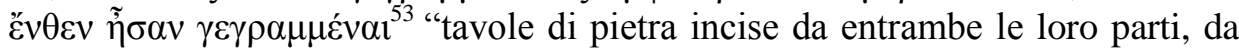
una parte e dall'altra erano scritte".

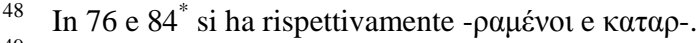

49 I codici compresi tra il 106 e il 370 hanno - $\rho \mu \varepsilon^{\prime} v o t$.

50 Non è da escludere l'ellissi dell'indicativo futuro ह̌бovtal e, dunque, la possibilità che i due participi rappresentino futuri perfetti.

51 La Vulgata (Biblia Sacra iuxta Vulgatam editionem, Stuttgart 1969) traduce al presente passivo: "bos tuus immoletur", come pure NETS, p. 166: "Your bull calf is butchered"; BRENTON, The Septuagint with Apocrypha, p. 269 opta per il futuro: "Thy calf shall be slain" (enfasi dell'autore), allo stesso modo MORTARI, Il Pentateuco, p. 855: "il tuo vitello sarà sgozzato".

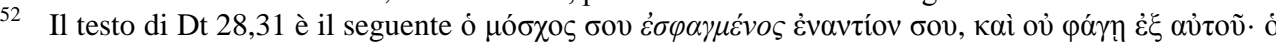

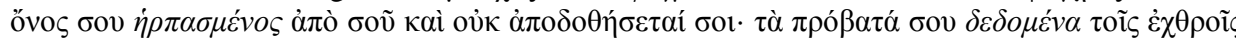

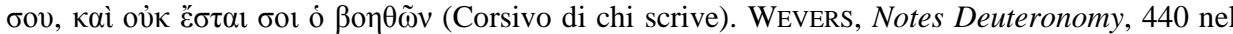
commentare il versetto osserva: "In each of three devastations the nominal clauses of MT have a passive participle as predicate, and the Greek translates each one literally without a linking verb. This creates a kind of staccato effect in Greek: 'your ox butchered before you, and you may not eat of it, etc.". L'analisi dei participi di Wevers come predicativi è un'alternativa possibile.

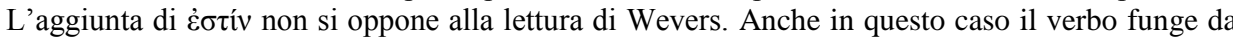
predicato verbale.

53 Evans, Verbal Syntax, p. 245 definisce la perifrasi piuccheperfetto indicativo; p. 254: suppletiva. 
Il primo participio è attributivo. Il codice 126(1475) omette $\tilde{\eta} \sigma \alpha v$. Si registra questa variante come esempio di ellissi dell'ausiliare.

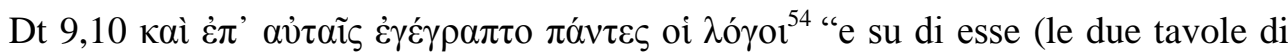
pietra) erano state scritte tutte le parole".

Il codice $\mathrm{V}$ (VIII) ha $\tilde{\eta} \sigma \alpha \nu ~ \gamma \varepsilon \gamma \rho \alpha \mu \mu \varepsilon \dot{\varepsilon}{ }^{\prime}$ or per $\dot{\varepsilon} \gamma \varepsilon \dot{\gamma} \gamma \alpha \pi \tau$. La corrispondenza aspettuale è rispettata.

\section{Predicato nominale vs forma verbale sintetica o inverso ${ }^{55}$}

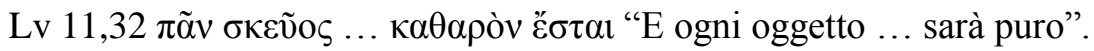

I codici 68' $[68(\mathrm{XV})+122(\mathrm{XV})]$ - 120' $[120(\mathrm{XI})+407$ (fine IX)] hanno

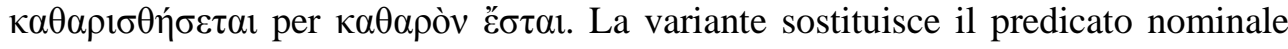
con un verbo denominativo corradicale della parte nominale ( $\kappa \alpha \theta \alpha \rho o ́ v)$. La forma verbale interpreta come passivo il costrutto predicativo, che di per sé esprime essenzialmente la qualità acquisita dal soggetto dopo essere stato immerso

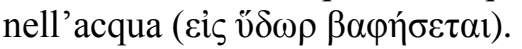

Casi del tutto simili, con stesso aggettivo e verbo, si registrano in: - 14,8 אaì

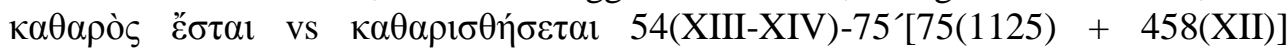

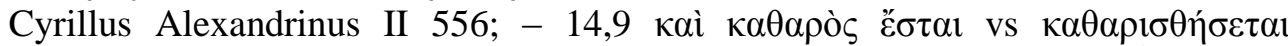

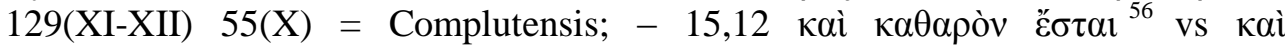

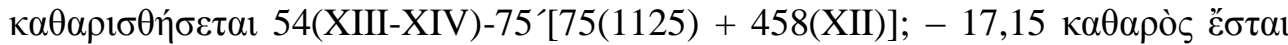

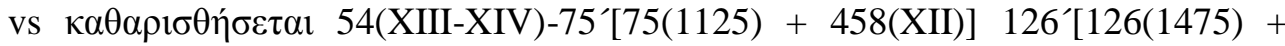
128(XIII)] 628' $[628(\mathrm{XIII})+630(\mathrm{X})] 646(\mathrm{XII})$.

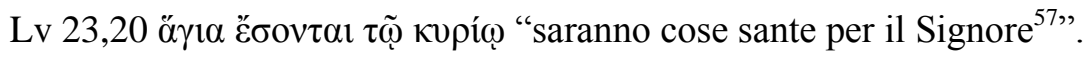

I testimoni del Gruppo $n^{-127}$ [54(XIII-XIV)-75(1125)-458(XII)-767(XIII-XIV)] hanno

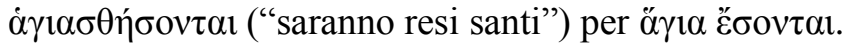

54 Evans, Verbal Syntax, p. 172 registra solo l'occorrenza del piuccheperfetto $\dot{\varepsilon} \gamma \varepsilon \dot{\gamma} \rho \alpha \pi \tau$.

55 Per completezza in questo paragrafo sono registrate le attestazioni di 'predicati nominali' con

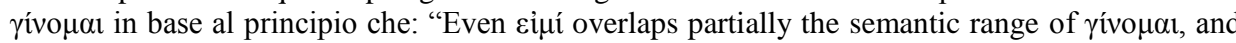
$\dot{\varepsilon} \gamma \varepsilon v o ́ \mu \eta \nu$ in the sense 'was' can arguably function as its aorist"; cf. Evans, Verbal Syntax, p. 223.

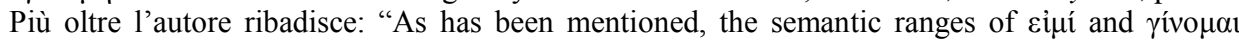
overlap when the latter's primary sense 'become' is weakened to 'be"' (225).

56 L'intero sintagma non ha corrispondenza nel Testo Masoretico.

57 Il predicato nominale si riferisce al capretto, agli agnelli e ai pani dei quali si parla in Lv 23,1920. 
Nm 5,28 $\dot{\alpha} \theta \omega \dot{\alpha} \alpha$ ह̌ $\sigma \tau \alpha l$ "(la donna) sarà innocente”.

La lezione ha come variante $\dot{\alpha} \theta \omega \omega \theta \eta \dot{\sigma \varepsilon \varepsilon \tau \alpha l ~ " s a r a ̀ ~ r i t e n u t a ~ i n n o c e n t e " ~ i n ~ V(V I I I) ~}$ $85(\mathrm{X})^{\mathrm{mg}}-321^{\text {'mg }}\left[321\right.$ (XIV)-346(1326)]-344(X) ${ }^{\mathrm{mg}}$ 319(1021).

La variante si avvale del verbo per esprime l'azione del riconoscimento dell'innocenza della donna al passivo: lei deve essere riconosciuta tale da altri.

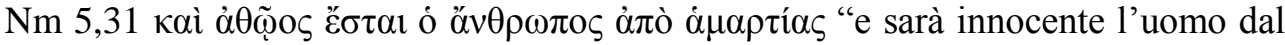
peccato".

In $30^{\prime}[30(\mathrm{XI}-\mathrm{XII})+730(\mathrm{XII})]$ si ha $\dot{\alpha} \theta \omega \omega \dot{\sigma} \sigma \varepsilon \tau \alpha$ per $\dot{\alpha} \theta \tilde{\omega} \mathrm{o} \varsigma^{\varepsilon} \sigma \tau \alpha \mathrm{l}^{58}$. Il predicato nominale esprime la qualità e la condizione soggettiva di innocenza dell'uomo geloso che "porrà sua moglie davanti al Signore, e il sacerdote applicherà a lei tutta questa legge"59. L'innocenza non è acquisita per il fatto o benché o se l'uomo compie l'azione descritta, ma si parla di lui come di un innocente che compie quell'azione. Il futuro medio $\dot{\alpha} \theta \omega \omega ́ \sigma \varepsilon \tau \alpha$ esprime tale condizione del soggetto e, concettualmente, si avvicina al predicato verbale.

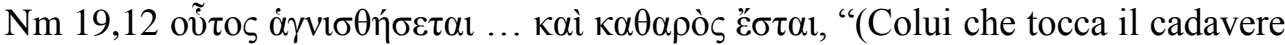
(v. 11)) costui sarà mondato ... sarà puro".

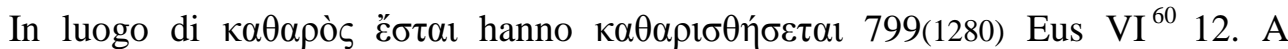

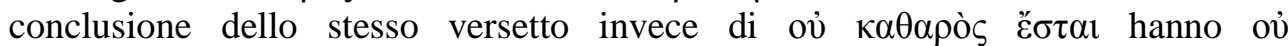
$\kappa \alpha \theta \alpha \rho \iota \sigma \theta \dot{\sigma} \sigma \varepsilon \tau \alpha \iota$ i codici 29(X-XI)-707(X-XI) 56’[56(1096) + 246(1195)] 85' ${ }^{\text {'mg }}[85(\mathrm{X})+$ 130(XII-XIII)]-321 'mg [321(XIV) + 346(1326)] 392(X) 319(1021) 799(1280).

L'adozione in entrambi i casi del futuro passivo $\kappa \alpha \theta \alpha \rho \iota \sigma \theta \eta ́ \sigma \varepsilon \tau \alpha \iota$ esplicita la

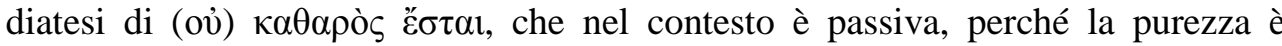

58 Il futuro medio di $⿱ \dot{\alpha} \theta \omega o ́ \omega$ non è regitrato nelle rispettive voci in H.G. SCOTT - H.ST. JONES - R. MCKenZIE, A Greek-English Lexicon (Oxford, 19499); A Supplement Edited by E.A. BARBER, with the Assistance of P. MAAS - M. SCHELLER - M.L. WEST, (Oxford, 1968); An English Lexicon, Revised Supplement Edited by P.G. Glare - A.A. ThOMPSON (Oxford, 1996); L. RoccI, Vocabolario greco-italiano (Città di Castello, $1966^{19}$ ); F. RodRÍGUEZ ADRADOS - E. GANGUTIA - J. LÓPEZ FACAL - C. SERRANO - P. BADENAS, Diccionario griego-español, Vol. I (Madrid, 1980); F. MONTANARI, Vocabolario della lingua greca (Torino, 1995); W. BAUER - K. ALAND - B. ALAND, A Greek-English Lexicon of the New Testament and other Early Christian Literature - Third Edition revised and edited by F.W. DANKER (Chicago - London, 2000); J. LUST - E. EYNIKEL - K. HAusPIE (LEH), Greek - English Lexicon of the Septuagint. Revised Edition (Stuttgart, 2003); T. MuRAOKA, A Greek-English Lexicon of the Septuagint (Louvain - Paris - Walpole, MA 2009). Il

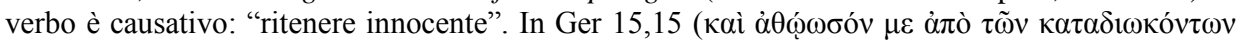
$\mu \varepsilon)$, più che il significato di "vendicare" (Brenton, LEH, Montanari), il verbo ha quello consueto causativo (NETS "and hold me guiltless before my persecutors") e quindi anche di "proteggere" (Muraoka).

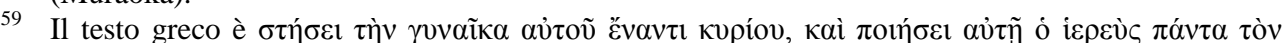

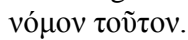

60 L'opera indicata è la Demonstratio evangelica di Eusebio di Cesarea (265-339 ca.). 
acquisita o no come conseguenza del rispetto o dell'inadempienza dell'azionenorma precedente.

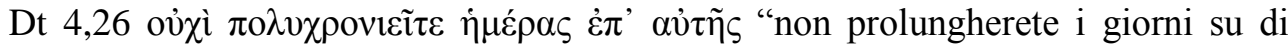
essa".

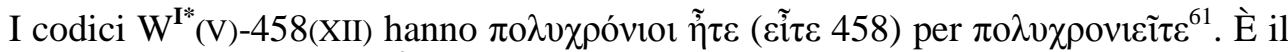
contesto di giuramento ${ }^{62}$ che deve aver indotto all'assunzione del congiuntivo $\tilde{\eta} \tau \varepsilon^{63}$. L'uso del congiuntivo con la particella negativa ov̉( $\left.\chi^{i}\right)$ si può ricollegare a quello del 'congiuntivo prospettivo' (dipendente) o può rientrare nell'uso del congiuntivo indipendente, lettura più probabile ${ }^{64}$. La distinzione tra il futuro e il congiuntivo, sul piano semantico, si può ricondurre a un'opposizione di "maggiore obiettività del futuro indicativo rispetto al congiuntivo" ${ }^{65}$, per cui la variante indirizza l'azione verso la soggettività. Contrariamente a quanto avviene altrove, inoltre, si passa dal verbo al predicato nominale, una scelta forse associata alla ricerca di enfasi.

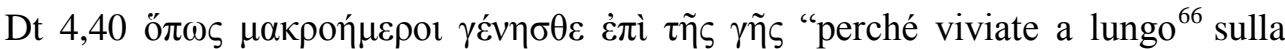
terra".

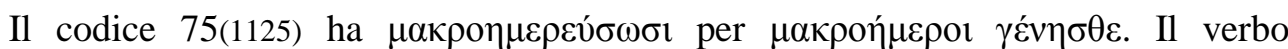

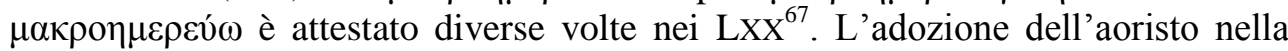

61 Il verbo è un neologismo dei Lxx come annota WeVERs, Deuteronomy, p. 82.

62 Mosè chiama a testimoniare il cielo e la terra

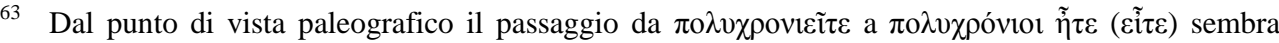
improbabile.

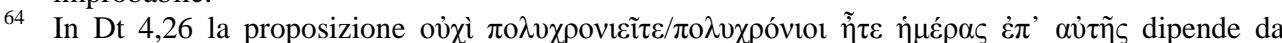
$\delta ı \mu \alpha \rho \tau$ $\rho \mu_{\alpha \iota} \ldots$... (ö $\left.\tau\right)$ o è indipendente? Le edizioni critiche la separano dal periodo precedente con un colon. Oltre che nell'edizione critica di Wevers la medesima punteggiatura appare in BRENTON, The Septuagint with Apocrypha, p. 236; H.B. SwETE (ed.), The Old Testament in Greek According to the Septuaginta, Vol. I: Genesis-IV Kings (Cambridge, $1909^{4}$, repr. 1925), p. 350; A. RAHLFS - R. HANHART (ed.), Septuaginta. Editio altera, Vol. I (Stuttgart, 2006), p. 292. Per il 'congiuntivo prospettivo' cf. L. TUSA MASSARO, Sintassi del greco antico e tradizione grammaticale (Palermo, 1993), pp. 162-163. Nella tradizione anglosassone si parla anche di Anticipatory Subjuntive, cf. H.W. SмYTH, Greek Grammar, revised by G.M. Messing (Cambridge, 1956, rist. 1976), $§ 1810$; in 1810a si legge: "This futural subjunctive is retained in Attic only in subordinate clauses". Tusa Massaro (162) ricorda che nell'Attico il congiuntivo prospettivo è accompagnato dalla particella ớ "solo nelle proposizioni secondarie in dipendenza di un tempo principale". Nel testo in esame non si ha né un tempo storico reggente né la presenza di ő̌v con il congiuntivo. La proposizione deve essere indipendente. Per dettagli di quest'uso 'indipendente' del congiuntivo (con negazione oủ) cf. JANNARIS, An Historical Greek Grammar, $\S 1921$ e soprattutto Appendix IV, 8-9.

65 N. BASILE, Sintassi storica del greco antico (Bari, 2001), p. 438 n. 193.

66 Non sembra proponibile tradurre: "siate longevi", più adeguata sarebbe la resa: "abbiate lunghi/molti giorni”". 
variante fa pensare alla ricerca di una corrispondenza aspettuale con la lezione originale. Ciò vuol dire che nel costrutto predicativo è la copula $\gamma \varepsilon ́ v \eta \sigma \theta \varepsilon$ a veicolare l'aspetto.

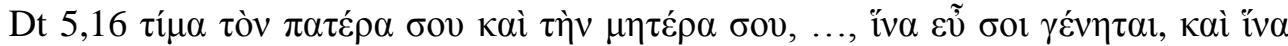

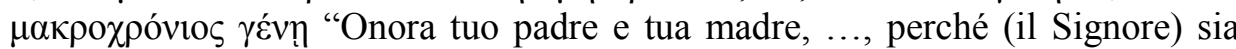
generoso verso di te e tu viva a lungo".

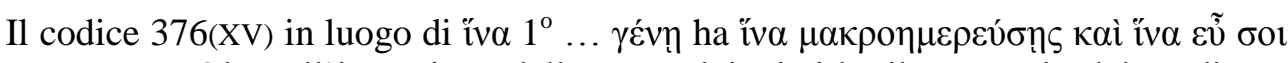
$\gamma \varepsilon \dot{\varepsilon} \eta \tau \alpha$. Oltre all'inversione delle proposizioni si ha il passaggio dal predicato

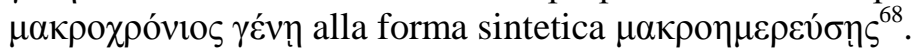

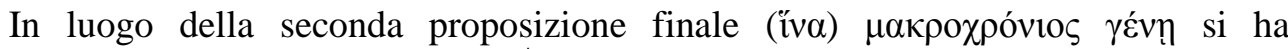

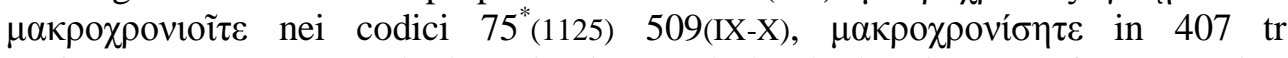
Or[igenes] IV 47. Entrambe le varianti sono al plurale, la prima è un futuro ottativo (desiderativo), la seconda un aoristo congiuntivo ${ }^{69}$.

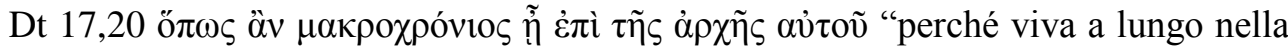
sua carica".

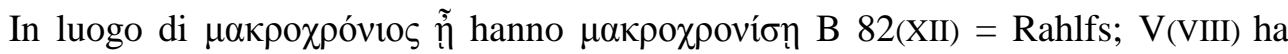

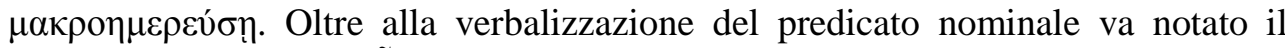

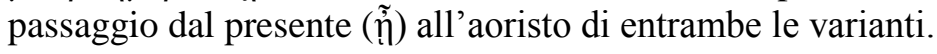

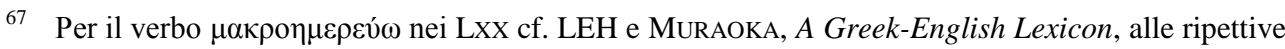
voci.

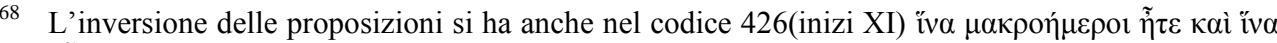

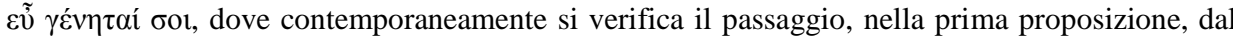
singolare al plurale.

69 Più frequenti le varianti con predicato nominale: $\mu \alpha \kappa \rho о \eta ́ \mu \varepsilon \rho o \imath ~ \tilde{\eta े \tau \varepsilon ~ 127(X)-767(X I I I-X I V) ; ~}$

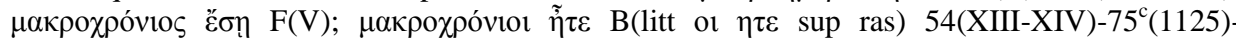

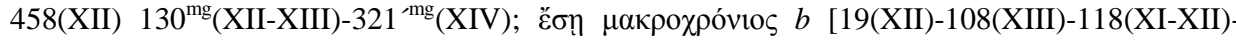
314(XIII)-537(XIII)] Eph 63. Per l'uso dell'ottativo dopo il congiuntivo cf. SMYTH, Greek Grammar, § 2199a, dove si dice che, in dipendenza da un tempo storico, soprattutto se a precedere è il congiuntivo, l'ottativo esprime, tra le due finalità, la meno immediata, intesa come conseguenza della precedente o come sola possibilità. Nel passo in esame non si ha un tempo storico ma l'ottativo può rispondere ugualmente alla relazione di conseguenza tra le due azioni. Tenuto conto, tuttavia, della datazione dei testimoni nei quali appare l'ottativo, sull'uso del modo appaiono illuminanti i seguenti dati. Nel capitolo Greek in Roman Empire, in G. HoRroKs, Greek. A History of the Language and its Speakers (London - New York, 1997), p. 88 l'autore scrive circa l'ottativo: "a mood increasingly associated with the literaly language". Nel capitolo Byzantine belles lettres (172), tra le peculiarità stilistiche di Michele Costantino Psello, Horroks nota: "He also employs a number of characteristic Byzantinisms, such as the free use of the optative as a 'marked' variant of the subjuntive even in non-past contexts". In seguito, nel discutere l'avvenuta confusione tra futuro indicativo e aoristo congiuntivo, osserva che in uno stesso scritto l'ottativo "also introduced as occasional 'learned' variants for both" (178). Enfasi dell'autore. La variante all'ottativo, in ultima analisi, può essere espressione di una scelta 'colta'. 


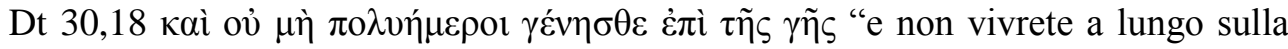
terra".

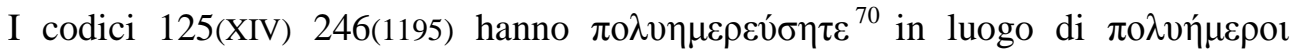
$\gamma \varepsilon \dot{v} \eta \sigma \theta \varepsilon$. Tra le due lezioni c'è corrispondenza aspettuale.

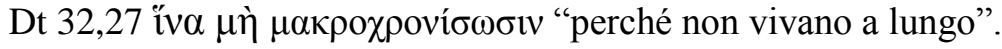

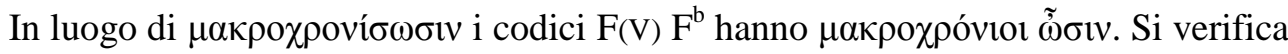
l'opposto della tendenza più frequente di sostituire il predicato nominale con la forma sintetica. Inoltre si passa dall'aoristo della forma sintetica al presente della copula del predicato nominale.

In margine si possono considerare altri due casi. 1) In Lv 14,36 yívouar non ha il valore di sìuí, ma si registra l'esempio a motivo della corrispondenza tra la costruzione predicativa e la variante verbale di forma finita (passiva). Il testo è $\kappa \alpha \grave{~}$

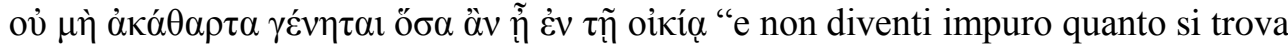
nella casa". I testimoni del Gruppo $n$ [54(XIII-XIV)-75(1125)-127(X)-458(XII)-

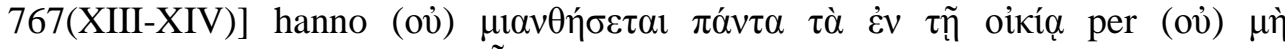

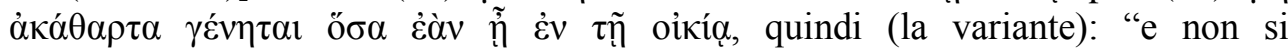
contamineranno tutte le cose che sono nella casa". La forma sintetica $\mu 1 \alpha v \theta \eta \dot{\sigma} \sigma \varepsilon \tau \alpha 1$

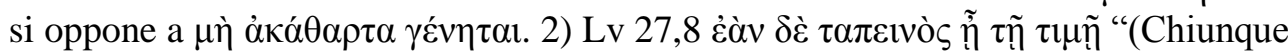

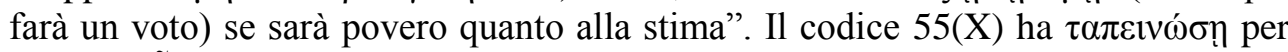
$\tau \alpha \pi \varepsilon$ vò $\hat{n ̃ n}_{\text {. Stando alla variante, il verbo denominativo-causativo } \tau \alpha \pi \varepsilon เ v o ́ \omega}$ sarebbe usato nel contesto intransitivamente, ma una lettura del genere non è convincente. Con molta probabilità la forma $\tau \alpha \pi \varepsilon เ v \omega \sigma \eta$ si deve a una scrittura errata di $\tau \alpha \pi \varepsilon เ v o \sigma \eta$, dunque allo scambio vocalico o $>\omega^{71}$.

\section{Conclusioni}

In primo luogo va rilevato che le varianti raccolte sono in grande maggioranza attestate in testimoni tardivi, tanto che talvolta, a causa del noto fenomeno diacronico della desistematizzazione del participio, in più di qualche caso - quando nella definizione dell'uso di un participio l'assenza di cỉní si può spiegare come ellissi (quindi uso perifrastico del participio) o semplicemente come segno d'uso

70 Nel codice 246 si legge - $\sigma \varepsilon \imath \tau \alpha$.

71 Il fenomeno è ricorrente. Casi che interessano forme verbali sono elencati in Á. URBÁN, "Bezae Codex Cantabrigiensis (D): intercambios vocálicos en Mateo”, CCO 5 (2008), pp. 349-350. Gentilmente l'autore mi ha segnalato ulteriori esempi che riguardano proprio $\tau \alpha \pi \varepsilon ı$ ó $\varsigma$ e derivati in testimoni del NT. In At 20,19 il codice 618(XII) ha $\tau \alpha \pi \varepsilon v \omega \varphi \rho о \sigma v v \eta$ per $\tau \alpha \pi \varepsilon \imath v o \varphi \rho \circ \sigma v v \eta$; in 2 Cor 10,1 il codice 2464 (IX) ha $\tau \alpha \pi \varepsilon 1 v \omega \varsigma$ per $\tau \alpha \pi \varepsilon 1 v o s$. Si verifica anche il contrario. In 2 Cor 11,7 il codice $\mathrm{F}^{*}(\mathrm{IX})$ ha $\tau \alpha \pi \mathrm{v}$ ov per $\tau \alpha \pi \varepsilon \mathrm{v} \omega v$. Va aggiunto che le varianti citate riguardano la fonologia e si tratta di casi di omonimia. 
del participio come forma verbale finita - si rimane incerti nell'interpretazione tra la costruzione perifrastica e l'uso 'finito' del participio. Tranne un caso (Es 1,910), tuttavia, la concordanza è osservata: non vi sono casi di mancata concordanza in genere, numero e caso ${ }^{72}$. Il numero di attestazioni raccolte conferma l'assunto iniziale di fondo, secondo cui nelle varianti si possono riflettere usi sintattici presenti in opere originali contemporanee ai testimoni nei quali appaiono.

Come si è constatato, sono le varianti al futuro (Gen 24,5.14, ma si consideri anche Es 3,18) e all'aoristo (tutte le attestazioni), a sollevare problemi interpretativi sintattici. Il futuro, tra $\mathrm{i}$ tempi, è quello che più di tutti ha una pluralità di espressione $^{73} \mathrm{e}$, nelle attestazioni, la perifrasi e il participio futuro mostrano qualche incertezza d'uso.

L'aoristo participio perifrastico ha un campo di impiego che va oltre l'indicativo ${ }^{74}$, ma nello stesso tempo, come si è rilevato in precedenza circa l'interpretazione perifrastica (con o senza ausiliare) in concorrenza con quella di verbo finito, riflette un uso fluttuante.

La discussione relativa al predicato nominale ha permesso di porre in rilievo il rapporto tra tale costruzione e un verbo finito, e di osservare come molte varianti verbali si spiegano principalmente per l'espressione della diatesi nell'azione.

Recibido / Received: 30/11/2012

Informado / Reported: 08/01/2013

Aceptado / Accepted: 14/01/2013

72 Non ci sono casi di participi assoluti "che preludono al participio presente attivo indeclinabile del greco moderno"; cf. L. CigneLLI - R. PIERRI, Sintassi di greco biblico (LXX e NT). Quaderno I.A Le concordanze (Jerusalem, 2003), § 14,5.

73 Cf. Jannaris, An Historical Greek Grammar, Appendix IV.

74 I casi elencati sono tutti in relazione all'indicativo ad eccezione del possibile aoristo participio imperativale in Es 32,27. Cf. inoltre note 32 e 42. 